\title{
Mortalidade infantil por causas evitáveis em municípios de fronteira e não fronteira
}

\author{
Infant mortality from preventable causes in border and \\ non-border cities
}

\author{
Paulo Cezar Rodrigues Martins ${ }^{1}$ (D), Elenir Rose Jardim Cury Pontes ${ }^{1}$ (D) \\ 'Universidade Federal de Mato Grosso do Sul (UFMS) - Campo Grande (MS), Brasil.
}

Como citar: Martins PCR, Pontes ERJC. Mortalidade infantil por causas evitáveis em municípios de fronteira e não fronteira. Cad Saúde Colet, 2020;28(2):201-210. https://doi.org/10.1590/1414-462X202028020096

\begin{abstract}
Resumo
Introdução: É grande o desafio de reduzir a mortalidade infantil por causas evitáveis no Brasil, dadas as desigualdades existentes no território. Objetivo: Estimar a taxa de mortalidade infantil por causas evitáveis e comparar os resultados entre os municípios de fronteira e de não fronteira, no estado de Mato Grosso do Sul. Método: Trata-se de um estudo ecológico. Foram estudados três grupos de municípios: Grupo 1 - municípios de fronteira contígua com área urbana no país vizinho; Grupo 2 - municípios de fronteira não contígua com área urbana no país vizinho; e Grupo 3 - municípios de não fronteira. Os dados foram obtidos a partir do Sistema de Informações sobre Mortalidade e do Sistema de Informações sobre Nascidos Vivos. Resultados: As taxas de mortalidade infantil por mil nascidos vivos, devido a causas evitáveis em 2004 e 2014, foram respectivamente: Grupo $1(21,8 / 11,29)$, Grupo 2 (24,68/14,7) e Grupo 3 $(14,3 / 7,23)$. A maior ocorrência dos óbitos ocorreu por causas relacionadas à inadequada atenção à mulher na gestação, parto, feto e ao recém-nascido. Conclusão: $O$ risco de óbito por causas evitáveis é maior nas crianças residentes nos municípios de fronteira, e isso deve ser considerado na elaboração das políticas e ações de saúde.
\end{abstract}

Palavras-chave: mortalidade infantil; causas de morte; estudos ecológicos.

\begin{abstract}
Introduction: There is a great challenge to reduce infant mortality from preventable causes in Brazil, given the inequalities that exist in the territory. Objective: To estimate the Infant Mortality Rate due to preventable causes and to compare the results between the border and non-border municipalities, in the State of Mato Grosso do Sul. Method: This is an ecological study. Three groups from cities were analyzed: Group 1 - contiguous cities with urban border in a neighboring country; Group 2 - non-contiguous cities with urban border in a neighboring country; e Group 3 - non-border cities. The data were obtained from Living Born Information System and Mortality Information System. Results: Infant mortality rates per 1,000 live births, by preventable causes in 2004 and 2014, were respectively: Group 1 (21.8 / 11.29), Group 2 (24.68 / 14.7) and Group 3 (14.3 / 7.23). The highest occurrence of deaths happened due to causes related to inadequate care to women during pregnancy, childbirth, fetus and the newborn. Conclusion: The risk of death due to preventable causes is higher in children living in border cities, and this should be considered in the elaboration of future health policies and actions.
\end{abstract}

Keywords: infant mortality; deaths cause; ecological studies.

\section{(c) (i)}

Este é um artigo publicado em acesso aberto (Open Access) sob a licença Creative Commons Attribution, que permite uso, distribuição e reprodução em qualquer meio, sem restrições desde que o trabalho original seja corretamente citado.

\footnotetext{
Trabalho realizado na Pós-graduação Saúde e Desenvolvimento da Região Centro Oeste, Universidade Federal de Mato Grosso do Sul (UFMS) - Campo Grande (MS), Brasil.

Correspondência: Paulo Cezar Rodrigues Martins. E-mail: pcrmpaulo@gmail.com

Fonte de financiamento: nenhuma.

Conflito de interesses: nada a declarar.

Recebido em: Mar. 05, 2018. Aprovado em: Jul. 16, 2019
} 


\section{INTRODUÇÃO}

A mortalidade infantil é um dos indicadores mais sensíveis à condição de vida de uma sociedade, pois reflete a situação de saúde do segmento mais vulnerável, que são os menores de 1 ano de idade. Taxas de mortalidade infantil (TMI) com valores elevados são indicativos de assistência precária em saúde e baixo desenvolvimento socioeconômico'.

A mortalidade de menores de 1 ano de idade representa $86 \%$ dos óbitos na infância. No Brasil, a taxa de mortalidade infantil reduziu 70\% entre 1990 (47,1 por mil nascidos vivos) e 2014 (14,1 por mil nascidos vivos). Até 1990, predominavam os óbitos no período pós-neonatal, onde era responsável por $51 \%$ das TMl; nas décadas seguintes, a maior predominância passou para o período neonatal, respondendo por $71 \%$ das TMI ${ }^{2}$.

As reduções observadas na mortalidade infantil não tiveram alcance uniforme no território nacional. As desigualdades socioeconômicas e a acessibilidade a bens e serviços estiveram associadas ao risco de uma criança não completar 1 ano de vida, fazendo com que ainda persistam taxas bem acima da média do Brasil ${ }^{3}$.

Os óbitos infantis decorrentes de causas evitáveis são delineados como aqueles que poderiam ser evitados, em parte ou total, em função de ações efetivas dos serviços de saúde que estejam acessíveis em um determinado local e época ${ }^{4-8}$. Apesar da ampliação dos serviços básicos de saúde, as taxas de mortalidade neonatal ainda apresentam resistências na redução $0^{9,10}$. Esses óbitos corresponderam a 68\% do total de óbitos de menores de 1 ano no Brasil, em 2014"11.

O objetivo do presente estudo foi estimar a frequência de óbitos de menores de 1 ano por causas evitáveis em 2004 e 2014, e comparar os resultados entre os municípios de fronteira e de não fronteira, no estado de Mato Grosso do Sul.

\section{MÉTODO}

Este é um estudo ecológico referente aos óbitos de menores de 1 ano em 2004 e 2014, que teve como unidade de análise, os municípios de linha de fronteira e de não fronteira do estado de Mato Grosso do Sul.

Os dados dos óbitos e de nascidos vivos foram extraídos dos Sistemas de Informações sobre Mortalidade (SIM) e Nascidos Vivos (SINASC) do Ministério da Saúde, por meio de consulta no site do Departamento de Informática do Sistema Único de Saúde (DATASUS).

Foram analisados os seguintes indicadores: números absolutos dos óbitos de menores de 1 ano de idade, taxas de mortalidade infantil (TMI) total e segundo causas de morte, variação percentual das TMls no período, TMI segundo a idade da criança e percentual dos óbitos evitáveis no total de óbitos.

O estudo analisou a magnitude da TMI, segundo as causas de óbitos evitáveis entre os grupos de municípios. A TMl é um indicador que estima o risco de morte no primeiro ano de vida e, sendo proxy das condições de vida da população de determinado espaço geográfico, identifica as desigualdades e vulnerabilidades existentes nas áreas.

Para o cálculo da taxa de mortalidade infantil (TMI), foi adotado o método direto. Segundo a Rede Interagencial de Informações para a Saúde (RIPSA), os cálculos diretos são recomendados quando o índice de cobertura e regularidade do SIM é igual ou superior a 80\% e a cobertura do SINASC igual ou superior a $90 \%{ }^{12}$. O estado de Mato Grosso do Sul atende aos critérios ora citados.

Para análise deste estudo, foram calculadas as TMIs, segundo a Lista de Causas de Mortes Evitáveis por intervenções do Sistema Único de Saúde (SUS) do Brasil|13,14.

As taxas foram assim classificadas:

- Causas evitáveis: (a) reduzíveis por ações de imunoprevenção; (b) reduzíveis por adequada atenção à mulher na gestação; (c) reduzíveis por adequada atenção à mulher no parto; (d) reduzíveis por adequada atenção ao recém-nascido; (e) reduzíveis por ações adequadas de diagnóstico e tratamento; (f) reduzíveis por ações adequadas de promoção à saúde, vinculadas a ações adequadas de atenção à saúde.

- Causas de mortes mal definidas;

- Causas de mortes não evitáveis (demais causas). 
O estado de Mato Grosso do Sul possui doze municípios localizados na linha de fronteira (Figura 1), em uma extensão de 1.520,5 km, sendo 1.128 km com o Paraguai e $392,5 \mathrm{~km}$ com a Bolívia. Esses municípios somaram 324.272 habitantes em 2014, cerca de 12\% da população do estado ${ }^{15}$.

Os municípios foram agrupados segundo as características geográficas do Projeto SIS-Fronteira no estado de Mato Grosso do Sul ${ }^{16}$ : Grupo 1 - municípios da linha de fronteira com áreas urbanas contíguas (Bela Vista, Coronel Sapucaia, Paranhos, Ponta Porã e Porto Murtinho); Grupo 2 - municípios da linha de fronteira sem área urbana contígua, mas com proximidade de área urbana (Antônio João, Aral Moreira, Caracol, Corumbá, Japorã e Mundo Novo) e Grupo 3 - demais municípios não localizados na linha de fronteira (Figura 1).

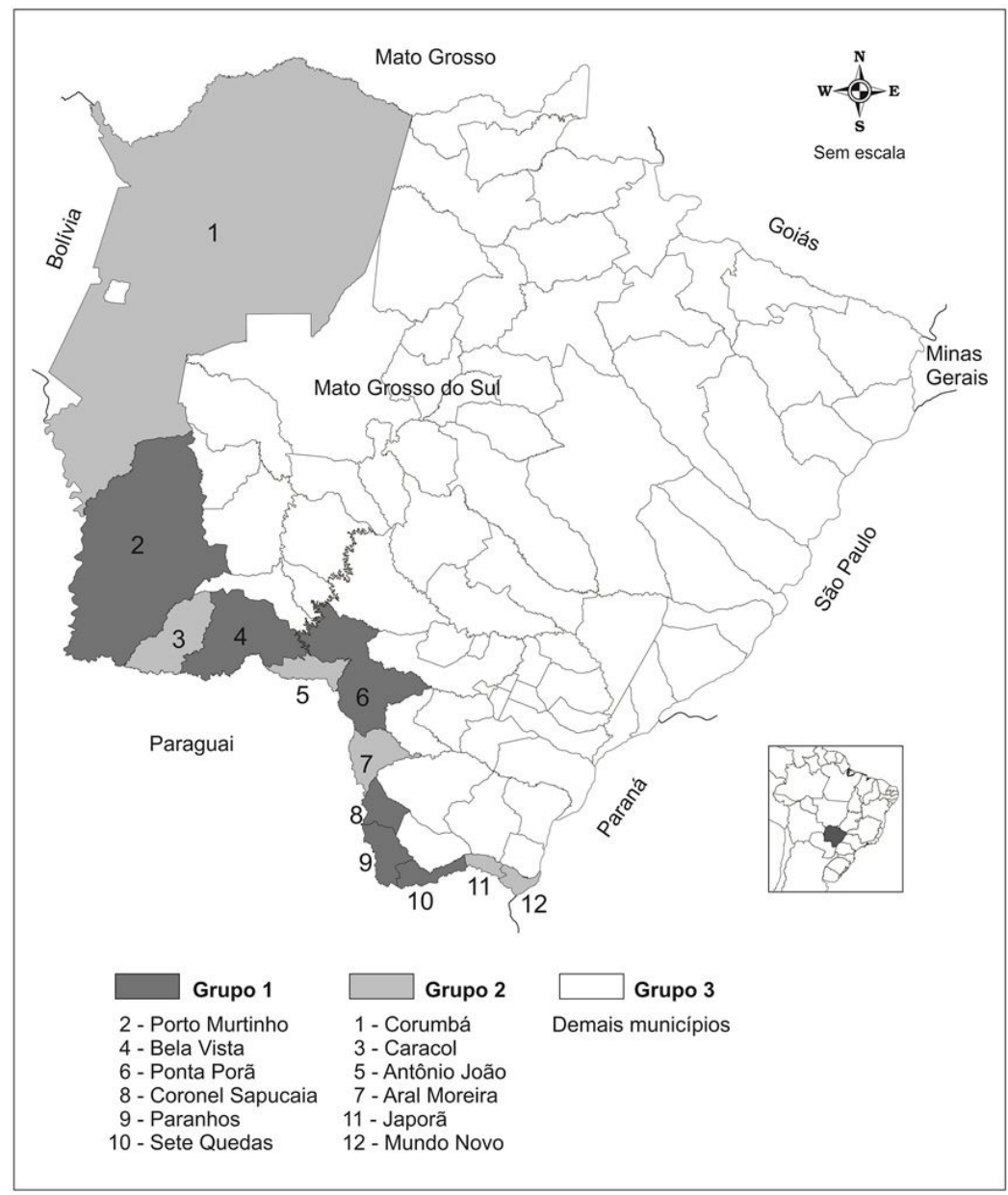

Figura 1. Municípios de Mato Grosso do Sul segundo grupos de estudo. Grupo 1 - municípios da linha de fronteira com áreas urbanas contíguas; Grupo 2 - municípios da linha de fronteira sem área urbana contígua, mas com proximidade de área urbana; e Grupo 3 - Demais municípios não localizados na linha de fronteira

Os resultados são apresentados na forma de tabelas e gráficos.

Este trabalho foi dispensado de avaliação ética de acordo com parecer do Comitê de Ética em Pesquisa com Seres Humanos da Universidade Federal de Mato Grosso do Sul, por se tratar de estudo que utiliza banco de dados não identificados e de acesso público.

\section{RESULTADOS}

No período em análise, o número de óbitos de menores de 1 ano relacionados a causas evitáveis obteve redução em todas as áreas de estudo, conforme resultados a seguir: no Grupo 1, de 81 para 35; no Grupo 2, de 78 para 43; e no Grupo 3, de 496 para 275 (Tabela 1). 


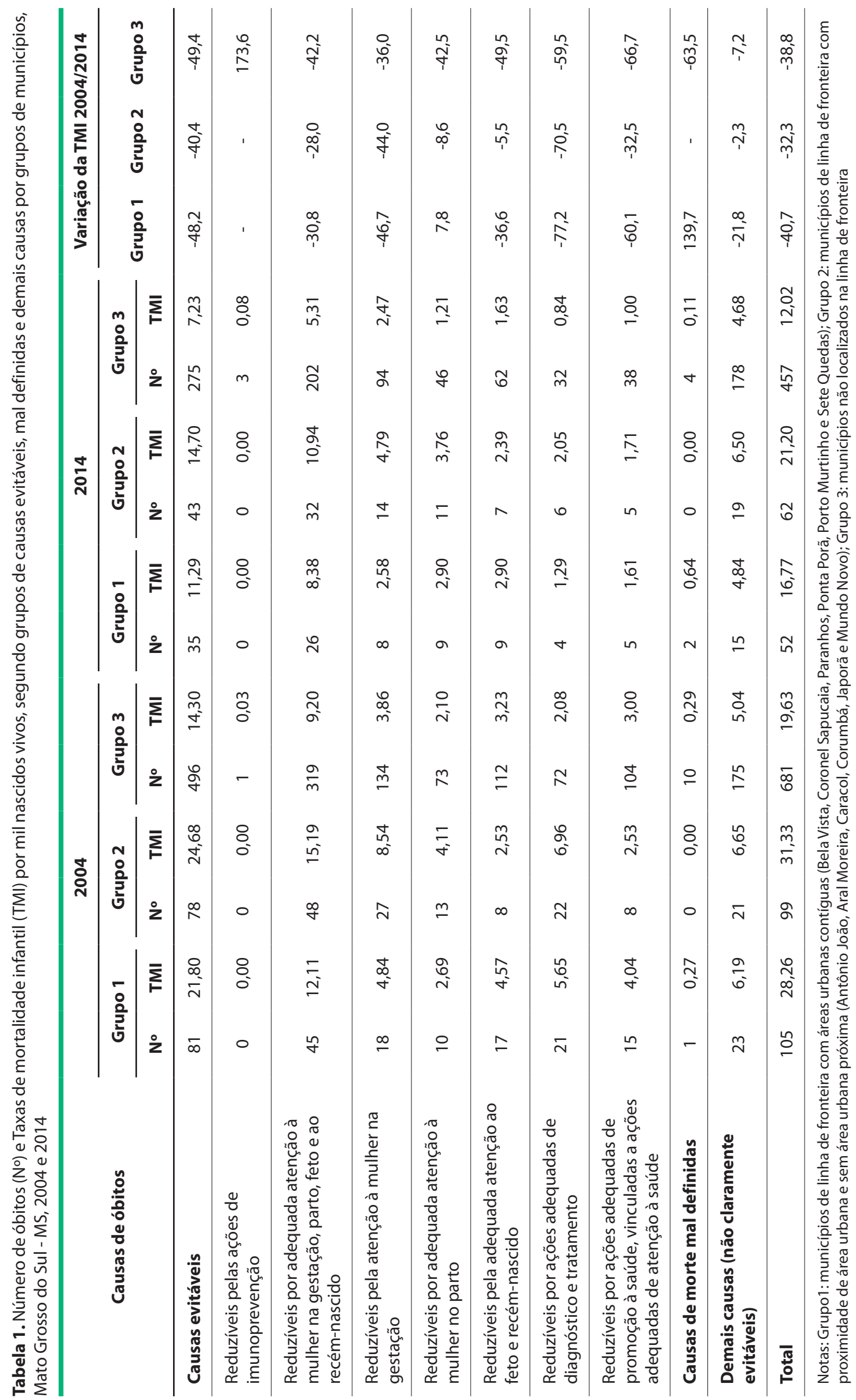


A TMI decorrente de causas evitáveis foi maior nos municípios do Grupo 2: 24,7 óbitos por mil nascidos vivos em 2004 e 14,7 óbitos por mil nascidos vivos em 2014. Em 2014, foi mais do que o dobro da TMI dos municípios do Grupo 3 (Tabela 1). Os óbitos por causas evitáveis por ações de imunização foram bem raros nos dois períodos. Em 2004, houve notificação de um caso nos municípios do Grupo 3 e, em 2014, três casos (Tabela 1).

Dentre os quatro grupos de óbitos por causas evitáveis, as reduzíveis por adequada atenção à mulher na gestação, parto, feto e ao recém-nascido apresentaram as maiores TMIs. Nos municípios do Grupo 2, foram observadas as maiores TMIs, sendo 15,19 óbitos por mil nascidos vivos e 10,94 óbitos por mil nascidos vivos respectivamente, para 2004 e 2014 (Tabela 1).

Os municípios do Grupo 2 destacam-se nas TMls das causas reduzíveis por adequada atenção à mulher na gestação, no período em análise (8,54 óbitos por mil nascidos vivos e 4,79 óbitos por mil nascidos vivos) e nas causas reduzíveis por adequada atenção à mulher no parto (4,11 óbitos por mil nascidos vivos e 3,76 óbitos por mil nascidos vivos) por apresentarem taxas bem superiores às demais áreas (Tabela 1).

Observou-se um aumento de 7,8\% nas TMIs de causas de óbitos relacionados à adequada atenção a mulher no parto nos municípios do Grupo 1, enquanto os municípios dos Grupos 2 e 3 tiveram redução no período analisado. As TMls resultantes das causas reduzíveis por adequada atenção ao feto e ao recém-nascido nos municípios do Grupo 1 são mais elevadas que as demais áreas estudadas, 4,57 óbitos por mil nascidos vivos e 2,90 óbitos por mil nascidos vivos em 2004 e 2014, respectivamente (Tabela 1).

O grupo de causas de óbitos reduzíveis por adequada atenção à mulher na gestação, parto, feto e ao recém-nascido teve a maior contribuição no total de óbitos nas três áreas de estudo. Em 2004, os municípios de não fronteira tiveram maior participação desse grupo de causas (64,3\%) em comparação aos municípios de fronteira (55,6\% e 61,5\%). Verificou-se que a contribuição dessas causas aumentou no ano de 2014 em todas as áreas, porém os percentuais se situaram bem mais próximos entre si (Figura 2).

Observou-se aumento do percentual de óbitos de causas reduzíveis por ações adequadas de promoção à saúde, vinculadas a ações adequadas de atenção à saúde nos municípios do Grupo 2, de 10,3\% em 2004 para 11,6\% em 2014. Nos demais grupos de municípios, houve redução no mesmo período (Figura 2).

As TMls por causas evitáveis, quando analisadas por idade da criança, mostram-se mais elevadas no período neonatal, concentrando-se mais nos óbitos de 0 a 6 dias de vida completos (neonatal precoce). Em 2004, nos municípios do Grupo 1, a TMI foi maior no período pós-neonatal, 9,1 óbitos por mil nascidos vivos (Tabela 2).

Tabela 2. Taxa de mortalidade infantil por mil nascidos vivos, de causas evitáveis, por componentes, segundo grupos de municípios, Mato Grosso do Sul - MS, 2004 e 2014

\begin{tabular}{clccc}
\hline \multirow{2}{*}{ Municípios } & Componentes & $\mathbf{2 0 0 4}$ & $\mathbf{2 0 1 4}$ & $\begin{array}{c}\text { Variação } \\
\text { percentual }\end{array}$ \\
\hline \multirow{2}{*}{ Grupo 1 } & Neonatal precoce & 8,9 & 5,8 & $-34,6$ \\
\cline { 2 - 5 } & Neonatal tardia & 3,8 & 3,2 & $-15,1$ \\
\cline { 2 - 5 } & Pós-neonatal & 9,1 & 2,3 & $-75,2$ \\
\hline \multirow{2}{*}{ Grupo 2 } & Neonatal precoce & 13,6 & 10,9 & $-19,6$ \\
\cline { 2 - 5 } & Neonatal tardia & 2,5 & 0,3 & $-86,5$ \\
\cline { 2 - 5 } & Pós-neonatal & 8,5 & 3,4 & $-59,8$ \\
\hline \multirow{2}{*}{ Grupo 3 } & Neonatal precoce & 7,3 & 4,1 & $-44,2$ \\
\cline { 2 - 5 } & Neonatal tardia & 2,4 & 1,2 & $-51,8$ \\
\cline { 2 - 5 } & Pós-neonatal & 4,6 & 2,0 & $-56,7$
\end{tabular}

Notas: Componentes da taxa de mortalidade infantil: Neonatal precoce: óbitos de 0 a 6 dias completos; Neonatal tardia: óbitos de 7 a 27 dias completos; Pós-neonatal: óbitos de 28 a 364 dias completos 

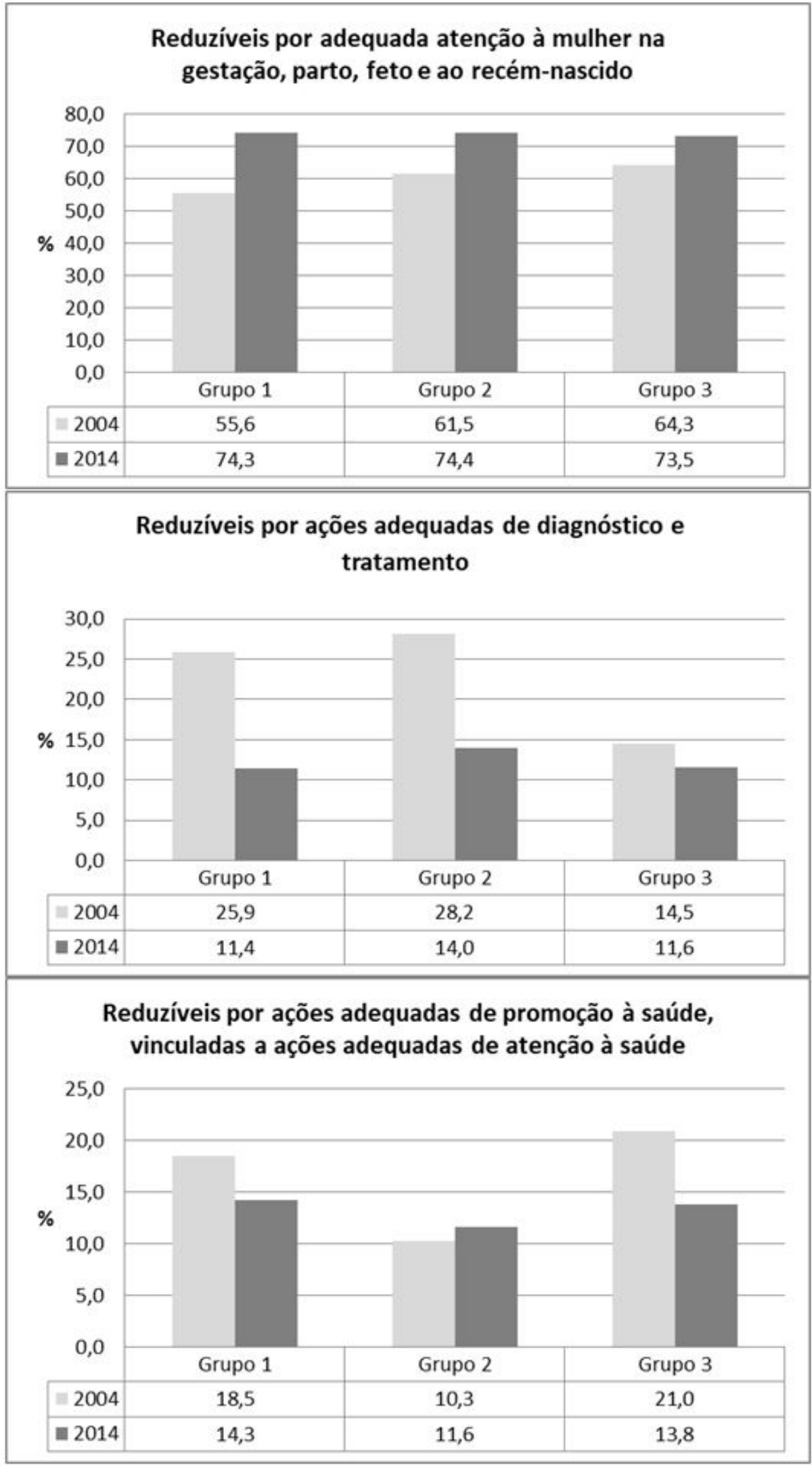

Figura 2. Distribuição percentual dos óbitos evitáveis de menores de 1 ano de idade, por grupos de causas, segundo grupos de municípios, Mato Grosso do Sul - MS, 2004 e 2014 
Nos municípios do Grupo 2, foram observadas as maiores TMls no período neonatal precoce, sendo 13,63 óbitos por mil nascidos vivos e 10,93 óbitos por mil nascidos vivos respectivamente, em 2004 e 2014. As TMls decorrentes de óbitos evitáveis no período neonatal tardia foram maiores nos municípios do Grupo 1 (3,8 óbitos por mil nascidos vivos em 2004 e 3,2 óbitos por mil nascidos vivos em 2014) (Tabela 2).

\section{DISCUSSÃO}

Os resultados encontrados neste estudo demonstram declínio da TMI nas áreas de fronteira e de não fronteira no período analisado, no entanto observam-se diferenciais entre as TMls, segundo as áreas estudadas. As características demográficas, econômicas e culturais nos municípios de fronteira influenciaram para que a probabilidade de morte de uma criança atingir seu primeiro ano de vida seja maior que a do grupo de municípios de não fronteira. Apesar dos avanços, muitas medidas ainda se fazem necessárias, como o desafio de reduzir as desigualdades socioeconômicas e regionais de saúde ${ }^{17-20}$.

No âmbito global, observam-se diferenças nas TMIs, principalmente quando se comparam os valores dos países desenvolvidos e em vias de desenvolvimento. Enquanto Portugal e Espanha já atingiram valores inferiores a 5 óbitos por mil nascidos vivos, México, Brasil e Chile apresentaram valores maiores em 2013, respectivamente 12,5, 12,30 e 7,10 óbitos por mil nascidos vivos ${ }^{21}$. No contexto nacional, observa-se que em 2014 a TMI do Brasil foi de 14,01 óbitos por mil nascidos vivos, enquanto nas regiões Sul e Norte foram respectivamente 10,7 e $17,7^{2}$.

No presente estudo, em 2014, a maior TMI (21,2 óbitos por mil nascidos vivos) foi observada no grupo de municípios de linha de fronteira sem área urbana contígua, seguida da TMI dos municípios de linha de fronteira com área urbana contígua $(16,8)$. É interessante notar que os valores das TMI dos países vizinhos no mesmo ano são altos, 18,1 no Paraguai e 31,5 na Bolívia ${ }^{22}$. Por outro lado, o grupo de municípios não localizados na linha de fronteira apresentou a menor TMI $(12,02)$, valor esse mais baixo e mais próximo ao do Brasil $(14,01)^{2}$. Isso demonstra que a proximidade com países com taxas piores refletiu negativamente nas TMI dos municípios fronteiriços, ratificando a influência de determinantes ambientais, demográficos e socioeconômicos.

A maior TMI relacionada à adequada atenção à mulher na gestação foi observada nos municípios da linha de fronteira com proximidade de área urbana e sem área urbana contígua. A baixa cobertura populacional da Estratégia Saúde da Família (ESF) em várias localidades no Brasil, as características geográficas e as desigualdades sociais podem influenciar o risco de óbito infantil no primeiro ano de vida ${ }^{23-25}$.

Foram observadas maiores taxas nas TMls decorrentes de causas reduzíveis por adequada atenção à mulher no parto e recém-nascido nos municípios de fronteira com área urbana contígua. O fato de o parto ser realizado em ambiente hospitalar não garante um procedimento de qualidade, tendo em vista que a maioria dos agravos do recém-nascido está associada à falta de eficiência na assistência obstétrica no país, principalmente quando são necessários serviços de terapia intensiva ${ }^{20,26,27}$.

As desvantagens que os municípios de fronteira apresentam em relação aos de não fronteira se tornam mais evidentes à medida que se exigem serviços de maior complexidade. A distância entre municípios dificulta a integração e a articulação dos serviços de saúde. A universalização do SUS ainda necessita de meios que garantam a acessibilidade aos municípios menos populosos e com maior vulnerabilidade socioeconômica ${ }^{28}$.

O grupo de causas reduzíveis por ações adequadas de diagnóstico e tratamento foi o que apresentou maiores reduções. O diagnóstico precoce, com precisão e adequada orientação, diminui os danos à saúde do recém-nascido, reduzindo assim o risco de óbito antes de completar 1 ano de vida. A criança deve receber maior atenção nas ações de saúde primária, permitindo diagnósticos precoces relacionados a causas determinantes ao crescimento, nutrição, desenvolvimento neuropsicomotor e orientação na prevenção de doenças e estímulos a hábitos saudáveis ${ }^{28,29}$. 
Observou-se um declínio nas TMIs decorrente de causas reduzíveis por ações adequadas de promoção à saúde, vinculadas a ações adequadas de atenção à saúde, no período de estudo em todos os grupos de municípios. Os agravos mais frequentes, como as doenças infecciosas intestinais e deficiências nutricionais, impactam mais o componente pós-neonatal e podem ser evitados com maior acesso a programas da ESF voltados à saúde materno e infantil, como a terapia de reidratação oral, maior cobertura de saneamento básico e programas de inclusão social ${ }^{20,30}$.

Ressalta-se também o número reduzido de óbitos por causas evitáveis devido a ações de imunização, o que demonstra o êxito do Programa Nacional de Imunização, que apresenta ampla cobertura vacinal nas áreas fronteiriças. Apesar disso, esses óbitos devem ser considerados eventos-sentinela?.

Os resultados da TMI decorrentes de causas evitáveis mostraram-se mais elevados no período neonatal precoce. Considerando que os maiores riscos dos óbitos neonatais são decorrentes, em grande parte, de assistência pré-natal inadequada, faz-se necessário não somente o olhar do acesso, mas principalmente na qualidade do serviço.

As TMls no período pós-neonatal foram mais elevadas nos municípios de fronteira com e sem área contígua. Óbitos infantis de 28 a 364 dias de vida, geralmente, estão associados ao desenvolvimento socioeconômico e à infraestrutura ambiental onde a criança vive, cresce e interage. Esses óbitos vêm perdendo participação no total da mortalidade em menores de $1 \mathrm{ano}^{31}$.

Cabe destacar que o número de pequenos eventos dos óbitos infantis nos municípios de pequeno porte populacional enseja uma análise diferencial na taxa de mortalidade infantil, assim podemos considerar esse fato como uma limitação deste estudo.

Este estudo permitiu verificar a redução na mortalidade infantil em todos os grupos de munícipios, porém foram observadas desigualdades na magnitude das taxas de mortalidade infantil e na variação da redução no período entre os municípios de fronteira e de não fronteira. O risco do óbito infantil por causas evitáveis mostra-se mais evidente nos municípios de fronteira. Foram observadas diferenças nas TMls entre municípios de contiguidade com área urbana, como o país vizinho, e os que não tinham contiguidade.

O desafio está em tratar dessas desigualdades por meio de ações que levem em consideração a região geográfica, as caraterísticas ambientais e a condição de vida da população. Medidas e ações genéricas sem considerar realidades distintas não serão eficazes, pois, muitas vezes, poderão acentuar as diferenças já existentes.

\section{REFERÊNCIAS}

1. Brasil. Ministério da Saúde. Pacto pela redução da mortalidade infantil no Nordeste e Amazônia Legal: 2009-2010 [Internet]. Brasília: Ministério da Saúde; 2010 [citado em 2017 Out 10]. Disponível em: http:// bvsms.saude.gov.br/bvs/publicacoes/pacto_reducao_mortalidade_infantil_nordeste.pdf

2. Brasil. Ministério da Saúde. Secretaria de Vigilância em Saúde. Departamento de Vigilância de Doenças e Agravos Não Transmissíveis e Promoção da Saúde. Saúde Brasil 2015/2016: uma análise da situação de saúde e da epidemia pelo vírus Zika e por outras doenças transmitidas pelo Aedes Aegypti. Brasília: Ministério da Saúde; 2016.

3. Gonçalves AC, Costa MDCN, Paim JS, Silva LMVD, Braga JU, Barreto M. Social inequalities in neonatal mortality and living condition. Rev Bras Epidemiol. 2013;16(3):682-91. http://dx.doi.org/10.1590/S1415790X2013000300012. PMid:24896281.

4. Fundo das Nações Unidas para a Infância. Levels \& trends in child mortality. Report 2015 estimates developed by the UN Inter-gency Group for Child Mortality Estimation [Internet]. New York: UNICEF/ WHO; 2015. [citado em 2017 Out 18]. Disponível em: https://www.unicef.org/publications/files/Child_ Mortality_Report_2015_Web_8_Sept_15.pdf

5. Brasil. Portal Brasil. ONU: Brasil cumpre meta de redução da mortalidade infantil. [Internet]. 2015 [citado em 2017 Set 19]. Disponível em: http://www.casacivil.gov.br/central-de-conteudos/noticias/2015/setembro/ onubrasil-cumpre-meta-de-reducao-da-mortalidade-infantil

6. Lassi ZS, Haider BA, Bhutta ZA. Community-based intervention packages for reducing maternal and neonatal morbidity and mortality and improving neonatal outcomes. Cochrane Database Syst Rev. 2010;11(11):CD007754. http://dx.doi.org/10.1002/14651858.CD007754.pub2. PMid:21069697. 
7. Moundzika-Kibamba JC, Nakwa FL. Neonatal mortality at Leratong Hospital. S. Afr. J. Child Health. 2018; 12(1):24-28. http://dx.doi.org/10.7196/sajch.2018.v12i1.1436.

8. Saleem S, McClure EM, Goudar SS, Patel A, Esamai F, Garces A, et al. A prospective study of maternal, fetal and neonatal deaths in low- and middle-income countries. B World Health Organ. 2014, 92(8):605-612.

9. Malta DC, Duarte EC, Escalante JJC, Almeida MF, Sardinha LMV, Macário EM, et al. Mortes evitáveis em menores de um ano, Brasil, 1997 a 2006: contribuições para a avaliação de desempenho do Sistema Único de Saúde. Cad. Saúde Pública. 2010;26(3):481-91. http://dx.doi.org/10.1590/S0102-311X2010000300006.

10. Brasil. Ministério da Saúde. Secretaria de Vigilância em Saúde. Departamento de Análise de Situação em Saúde. Saúde Brasil 2013: uma análise da situação de saúde e das doenças transmissíveis relacionadas à pobreza. Brasília: Ministério da Saúde; 2014.

11. Datasus. Tecnologia da Informação a Serviço do SUS [Internet]. 2017 [citado em 2017 Out 16]. Disponível em: http://tabnet.datasus.gov.br/cgi/deftohtm.exe?sim/cnv/inf10ms.def

12. Rede Interagencial de Informações para a Saúde. Indicadores básicos para a saúde no Brasil: conceitos e aplicações. 2. ed. Brasília: OPAS; 2008.

13. Malta DC, Duarte EC, Almeida MF, Dias MAS, Morais OL No, Moura L, et al. Tabela Brasileira de Causas de Mortes Evitáveis por Intervenções do Sistema Único de Saúde. Epidemiol Serv Saude. 2007;16(4):233-44.

14. Malta DC, França E, Abreu DX, Oliveira H, Monteiro RA, Sardinha LMV, et al. Nota Técnica: Atualização da lista de causas de mortes evitáveis ( 5 a 74 anos de idade) por intervenções do Sistema Único de Saúde do Brasil [Internet]. Epidemiol Serv Saúde. 2011;20(3):409-412 [citado em 2017 Set 11]. Disponível em: http://scielo.iec.pa.gov.br/pdf/ess/v20n3/v20n3a16.pdf.

15. Mato Grosso do Sul. Secretaria de Estado de Meio Ambiente e Desenvolvimento Econômico (SEMADE). Perfil Estatístico de Mato Grosso do Sul 2015: ano base 2015. Campo Grande: SEMADE; 2016.

16. Tamaki EM, Ferraz AF, Pontes ERJC, Cazola LHDO, Ajalla MEA, Pícoli RP, et al. O Projeto SIS-fronteira no Estado de Mato Grosso do Sul. In: Souza ML editor. A saúde e a inclusão social nas fronteiras. Florianópolis: Boiteux; 2008.

17. Arrué AM, Neves ET, Silveira A, Pieszak GM. Caracterização da morbimortalidade de recém-nascidos internados em unidade de terapia intensiva neonatal. Rev Enferm UFSM. 2013;3(1):86-92. http://dx.doi. org/10.5902/217976925947.

18. Oliveira BRG, Viera CS, Furtado MCC, Mello DF, Lima RAG. Perfil de morbidade de crianças hospitalizadas em um hospital público: implicações para a Enfermagem. Rev Bras Enferm. 2012;65(4):586-93. http:// dx.doi.org/10.1590/S0034-71672012000400006. PMid:23258677.

19. Oliveira RR, Costa JR, Mathias TAF. Hospitalizações de menores de cinco anos por causas evitáveis. Rev Lat Am Enfermagem. 2012;20(1):135-42. http://dx.doi.org/10.1590/S0104-11692012000100018. PMid:22481731.

20. Victora CG, Aquino EML, Leal MC, Monteiro CA, Barros FC, Szwarcwald CL. Maternal and child health in Brazil: progress and challenges. Lancet. 2011;377(9780):1863-76. http://dx.doi.org/10.1016/S01406736(11)60138-4. PMid:21561656.

21. Observatório Ibero-Americano de Políticas e Sistemas de Saúde. Matriz de Indicadores [Internet]. 2019 [citado em 2019 mai 15]. Disponível em: http://oiapss.icict.fiocruz.br/matriz.php?ling=2

22. Instituto Suramericano de Gobierno em Salud. Tasa de mortalidad infantil - CEPAL [Internet]. 2019 [citado em 2019 mai 23]. Disponível em: http://isags-unasur.org/dados-2-2-2-2/

23. Ribeiro AM, Guimarães MJ, Lima MC, Sarinho SW, Coutinho SB. Fatores de risco para mortalidade neonatal em crianças com baixo peso ao nascer. Rev Saude Publica. 2009;4(2):246-55. http://dx.doi.org/10.1590/ S0034-89102009005000004. PMid:19225697.

24. Fundo das Nações Unidas para a Infância. Mortalidade de crianças: infância ainda vulnerável [Internet]. New York: UNICEF/WHO; 2015 [citado em 2017 Set 10]. Disponível em: https://www.unicef.org/brazil/pt/ Pags_008_019_Mortalidade.pdf

25. Ceccon RF, Bueno ALM, Hesler LZ, Kirsten KS, Portes VM, Viecili PRN. Mortalidade infantil e Saúde da Família nas unidades da Federação brasileira, 1998-2008. Cad Saude Colet. 2014;22(2):177-83. http:// dx.doi.org/10.1590/1414-462X201400020011.

26. Brasil. Ministério da Saúde. Secretaria de Vigilância em Saúde. Departamento de Análise de Situação de Saúde. Saúde Brasil 2011: uma análise da situação de saúde e a vigilância da saúde da mulher. Brasília: Ministério da Saúde; 2012.

27. Oliveira EFV, Gama SGN, Silva CMFP. Gravidez na adolescência e outros fatores de risco para mortalidade fetal e infantil no município do Rio de Janeiro, Brasil. Cad Saude Publica. 2010;26(3):567-78. http://dx.doi. org/10.1590/S0102-311X2010000300014. PMid:20464075. 
28. Brasil. Ministério da Saúde. Programa Nacional de Melhoria do Acesso e da Qualidade da Atenção Básica (PMAQ) [Internet]. Brasília: Ministério da Saúde; 2012 [citado em 2017 Set 18]. Disponível em: http://dab. saude.gov.br/portaldab/cidadao_pmaq2.php

29. Oliveira BRG, Viera CS, Collet N, Lima RAG. Causas de hospitalização no SUS de crianças de zero a quatro anos no Brasil. Rev Bras Epidemiol. 2010;13(2):268-77. http://dx.doi.org/10.1590/S1415-790X2010000200009.

30. Malta DC, Sardinha LMV, de Moura L, Lansky S, Leal Mdo C, Szwarcwald CL, et al. Atualização da lista de causas de mortes evitáveis por intervenções do Sistema Único de Saúde do Brasil. Epidemiol Serv Saude. 2010;19(2):173-6.

31. Rasella D, Aquino R, Santos CAT, Paes-Sousa R, Barreto ML. Effect of a conditional cash transfer programme on childhood mortality: a nationwide analysis of Brazilian municipalities. Lancet. 2013;382(9886):57-64. http://dx.doi.org/10.1016/S0140-6736(13)60715-1. PMid:23683599. 